\title{
Multiple Giant Splenic Artery Aneurysms with Hypersplenism and Portal Hypertension: A Case Report
}

\author{
Zia Ur Rehman, MBBS, FCPS, ChM Vascular and Endovascular Surgery
}

Multiple giant splenic artery aneurysms $(>10 \mathrm{~cm}$ in diameter) are rare. We report a patient presenting with these aneurysms who had also associated portal hypertension and massive splenomegaly. Almost all the aneurysms were sealed by endovascular means at the first stage. Later, for the residual aneurysms at splenic hilum and enlarged spleen, splenectomy was performed. She made an uneventful recovery.

Keywords: splenic artery, giant aneurysm, endovascular treatment

\section{Introduction}

Giant splenic artery aneurysms (SAAs) measuring more than $10 \mathrm{~cm}$ in diameter are rare. ${ }^{1)}$ Multiple giant SAAs associated with portal hypertension and hypersplenism are even more rare. We report a case in which patient presented with abdominal pain and was found to have multiple SAAs and portal hypertension. She was treated successfully using both endovascular and surgical options.

\section{Case Report}

A 22-year-old female reported left upper abdominal pain for one month. It was mild to moderate and not associated

Section of Vascular Surgery, Department of Surgery, Agha Khan University Hospital, Karachi, Pakistan

Received: February 4, 2019; Accepted: March 8, 2019

Corresponding author: Zia Ur Rehman, MBBS, FCPS, ChM Vascular and Endovascular Surgery. Section of Vascular Surgery, Department of Surgery, Agha Khan University Hospital, Stadium Road, Karachi 74800, Pakistan

Tel: +92-32-12039951, Fax: +92-21-34932095

E-mail: ziaur.rehman@aku.edu

(cc) BY-NC-SA (C2019 The Editorial Committee of Annals of Vascular Diseases. This article is distributed under the terms of the Creative Commons Attribution License, which permits use, distribution, and reproduction in any medium, provided the credit of the original work, a link to the license, and indication of any change are properly given, and the original work is not used for commercial purposes. Remixed or transformed contributions must be distributed under the same license as the original. with any gastrointestinal symptoms. It was relieved by analgesics. She did not give history suggestive of tuberculosis or pancreatitis. Abdominal examination revealed splenomegaly and a pulsatile abdominal mass. On abdominal ultrasound, she was found to have multiple SAAs and massive splenomegaly. The findings were confirmed by abdominal computed tomography. It did show multiple SAAs, the largest one was measuring $10 \mathrm{~cm}$ (Fig. 1). It also showed a thrombosed portal vein with cavernous formation and splenomegaly measuring $231.9 \mathrm{~mm}$. The patient had low platelets and leukocyte counts. Preoperative serological markers were negative for hepatitis $\mathrm{B}$ and $\mathrm{C}$ viruses. The risks and benefits of intervention were discussed with her. She was admitted for the angioembolization of SAAs (Fig. 2). Initially a C1 catheter was used to cannulate the hepatic artery; an exchange wire was

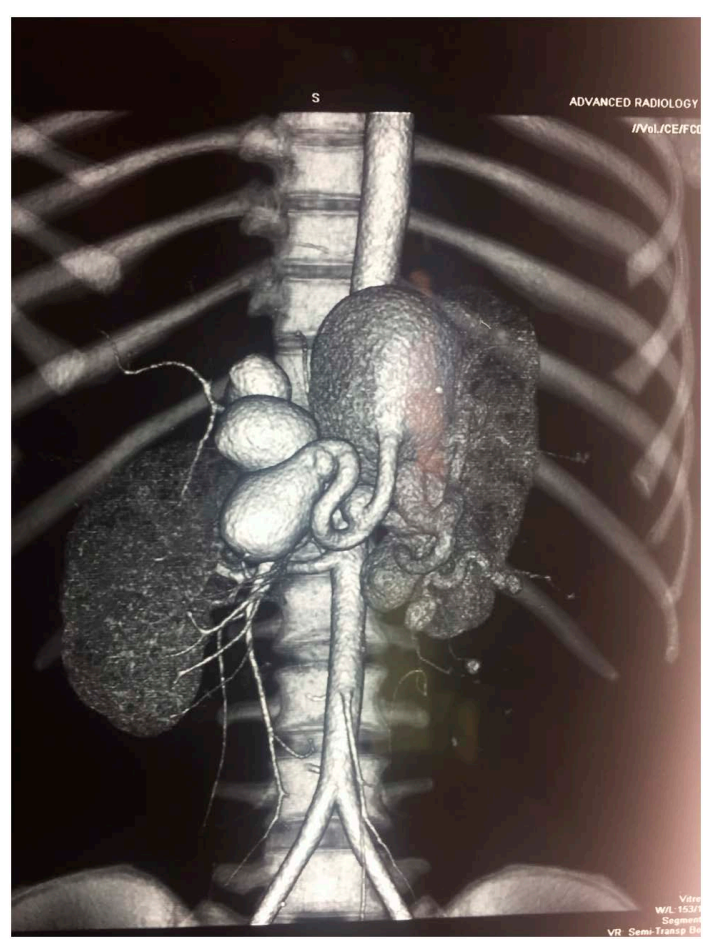

Fig. 1 Computed tomography showing multiple splenic artery aneurysms. 


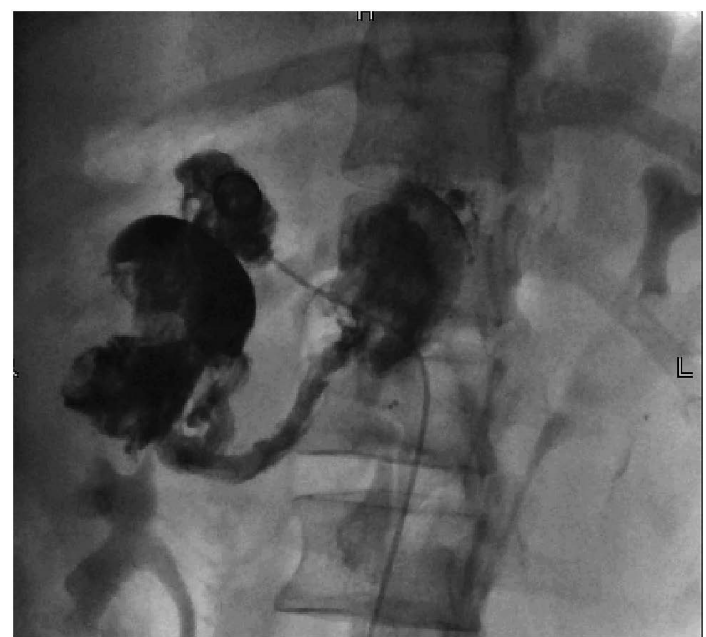

Fig. 2 Multiple splenic artery aneurysms being embolized by glue and coils.

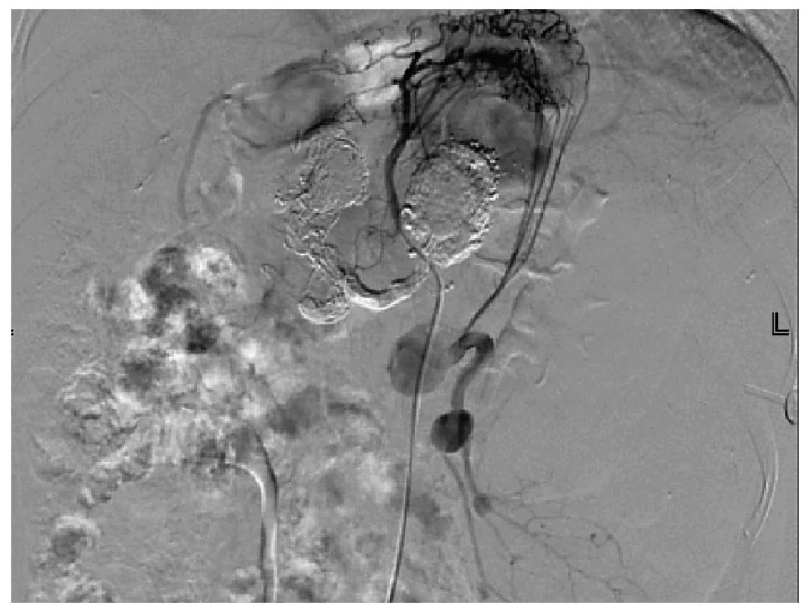

Fig. 3 Visceral angiogram showing almost total occlusion of multiple splenic artery aneurysms. The two filling were from short gastric arteries at the splenic hilum. The largest one was measuring $23 \mathrm{~mm}$ in diameter.

parked in the right hepatic artery. An $8 \times 40 \mathrm{~mm}$ balloon was kept in the celiac artery to occlude the splenic artery origin. A C1 catheter and microcatheter were used to cannulate the splenic artery as distally as possible. Glue was used to embolize the aneurysms. After $24 \mathrm{~h}$, a check angiogram showed the embolized main splenic artery having persistent flow into the aneurysms. There were two gastric arteries supplying gastric fundus and forming multiple short gastric arteries to supply the spleen poles. The lower pole artery showed multiple small aneurysms, the largest measuring $23 \times 18 \mathrm{~mm}$. The splenic artery was again cannulated and completely embolized with Glubran 2 Glue (N butyl 2 Cyanoacrylate and MS) (GEM Srl, Viareggio, Italy). No residual flow was noted in the splenic artery. However, on the final angiographic run, the short gastric arteries were still filling the aneurysm in the splenic hilum.
The aneurysm measured $23 \mathrm{~mm}$ (Fig. 3). For the residual aneurysm and hypersplenism, she underwent surgery with splenectomy and ligation of the residual aneurysm through an upper midline incision. The liver had a normal color and texture with no ascites and little inflammation. The lesser sac was entered by dividing the gastrocolic ligament. Previously embolized aneurysms were embedded in the mass of the pancreas. There were residual true aneurysms noted around the splenic hilum. Multiple large varicosities were divided in the splenic hilum along with the vessels of splenic hilum. Later splenocolic, linorenal, and splenodiaphragmatic ligaments were divided and the spleen was brought in the midline. Splenectomy was performed with less than $100 \mathrm{ml}$ of blood loss. The patient had a smooth recovery. Her platelets and white blood cell counts improved post operatively. The final spleen histopathology showed features of congestive splenomegaly with the surface of spleen and hilum showing congested and dilated vessels. An aneurysm near the splenic hilum was looking true since it was fusiform, involving all the walls and with no surrounding inflammation. We do not have final histopathology. No organism was cultured form the specimen.

\section{Discussion}

SAA has an incidence of 0.2 to $2 \% .{ }^{2)}$ It is the most common of visceral artery aneurysms compromising $50-75 \%$ of all cases. In addition, they are the third most frequent intra-abdominal aneurysm, following abdominal aorta and iliac artery aneurysms. ${ }^{3)}$ The annual risk of rupture is $2-10 \%$. They are more prevalent in females $(4: 1)$. They present as clinical emergencies in $22 \%$ of the cases with an overall mortality rate of $8.5 \%{ }^{4}$ ) Most small SAAs (up to $2 \mathrm{~cm}$ ) are asymptomatic and diagnosed incidentally. ${ }^{5)}$ In contrast, most large SAAs $(5 \mathrm{~cm})$ are symptomatic and can result in complications. $\left.{ }^{6}\right)$ A giant SAAs is more than $10 \mathrm{~cm}$ in size. There are many case reports and case series describing giant SAAs but very few describing multiple SAAs. The risk of rupture in giant SAAs is as high as $28 \%,{ }^{7)}$ with a mortality rate of up to $40 \% .{ }^{8)}$

Our intention was to seal all aneurysms by endovascular means to save the patient from surgical intervention. We were expecting that by reduction of size of aneurysms, the compression effect on the portal system will decrease. We were not able to completely occlude all the aneurysms, especially one in the splenic hilum which was filling through short gastric arteries. One stage intervention with ligation of aneurysms and splenectomy can be an option. Beksac and Karakoc reported surgical treatment of a patient with multiple SAAs in which the patient presented with portal hypertension and splenomegaly. ${ }^{9}{ }^{9} \mathrm{~A}$ two-staged procedure provided a more controlled situation, as noted by the 
minimal blood loss in our case.

This patient was having non-cirrhotic portal hypertension. Her preoperative liver function tests were normal. Preoperative serological markers were also negative for hepatitis B and C viruses. Intra-operatively her liver was looking normal. Her portal hypertension was due to the compression effects of multiple giant SAAs. Khan et al. also reported a patient in 2016 with a giant SAAs which led to non-cirrhotic portal hypertension in that patient. ${ }^{10)}$

Multiple SAAs can present in patients with collagen vessel or connective tissue disease. Our patient had no particular symptoms related to Marfan's syndrome or other connective tissue diseases. There was no familial history of aneurysms. Performing specific genetic and collagen testing to identify selective patients may be useful.

This case highlights that, with the help of both endovascular and surgical means, patients with multiple SAAs can be managed successfully.

\section{Conclusion}

Patient with multiple SAAs can be treatable using both endovacular and surgical interventions together safely.

\section{Disclosure Statement}

The author has no conflict of interest.

\section{References}

1) Huang IH, Zuckerman DA, Matthews JB. Occlusion of a giant splenic artery pseudoaneurysm with percutaneous thrombin-collagen injection. J Vasc Surg 2004; 40: 574-7.

2) Pulli R, Dorigo W, Troisi N, et al. Surgical treatment of visceral artery aneurysms: a 25 -year experience. J Vasc Surg 2008; 48: 334-42.

3) Miao YD, Ye B. Intragastric rupture of splenic artery aneurysms: three case reports and literature review. Pak J Med Sci 2013; 29: 656-9.

4) Ferrero E, Ferri M, Viazzo A, et al. Visceral artery aneurysm, an experience of 32 cases in a single center: treatment from surgery to multilayer stent. Ann Vasc Surg 2011; 25: 923-35.

5) Rathod J, Taori K, Dhomne S, et al. Endovascular embolisation of giant ruptured proximal splenic arterial pseudoaneurysm using microcoils \& glue: case report. Surg Sci 2011; 2: 290-3.

6) Akbulut S, Otan E. Management of giant splenic artery aneurysm: comprehensive literature review. Medicine (Baltimore) 2015; 94: e1016.

7) Jamsheer NS, Malik N. Ruptured splenic artery aneurysm. Ann Saudi Med 2001; 21: 340-1.

8) Karaman K, Onat L, Șirvancı M, et al. Endovascular stent graft treatment in a patient with splenic artery aneurysm. Diag Interv Radiol 2005; 11: 119-21.

9) Beksac K, Karakoc D. Multiple giant splenic artery aneurysm causing sinistral (left-sided) portal hypertension. Case Rep Gastrointest Med 2016; 2016: 6278452.

10) Khan A, Ayub M, Haider I, et al. Coexisting giant splenic artery and portal vein aneurysm leading to non-cirrhotic portal hypertension: a case report. J Med Case Reports 2016; 10: 270 . 